\title{
Impactos da prática de exercícios físicos na qualidade de vida da pessoa idosa: revisão integrativa
}

\author{
Impacts of practice of physical exercises on the quality of life of elderly people: integrative \\ review
}

\author{
Impactos de la práctica de los ejercicios físicos en la calidad de vida de las personas \\ mayores: revisión integrativa
}

Genivalda Santos da Silva ${ }^{1}$, Núbia Cecília da Conceição dos Santos ${ }^{1}$, Thais dos Santos Batista ${ }^{1}$, Jaqueline Santos da Conceição", Cinthia Ravena Figueiredo Lira¹, Luna Vitória Cajé Moura1*.

\section{RESUMO}

Objetivo: Dissertar sobre o que tem sido publicado no meio científico, sobre a relação entre as práticas de exercícios físicos por pessoas idosas e sua qualidade de vida e saúde. Métodos: Trata-se de uma revisão integrativa da literatura, tendo como base a coleta de informações nos bancos de dados eletrônicos, utilizando a metodologia cientifica exploratória. Utilizando critérios de inclusão e exclusão: Sucederam, períodos, idiomas, artigos, textos completos. Resultados: Após filtragem com base nos critérios de inclusão e exclusão, treze artigos restaram, respondendo aos objetivos do presente estudo. Foram, a partir destes apresentados diversos impactos da prática de exercícios físicos na saúde e qualidade de vida da pessoa idosa, e evidenciados nas categorias e subcategorias que há impactos positivos na saúde mental e na saúde física das pessoas idosas, reverberando diretamente em sua qualidade de vida. Considerações finais: Concluiu-se que são perceptíveis os benefícios da prática de exercícios físicos assistidos, e conforme a tolerância de cada idoso, na saúde e qualidade de vida da pessoa idosa e configuram-se também como principal fonte promotora do bem-estar físico, psíquico e social para este público.

Palavras-chave: Exercício físico, Qualidade de vida, Idoso.

\begin{abstract}
Objective: To discover what has been published in the scientific community, about the relationship between the practice of physical exercise by elderly people and their quality of life and health. Methods: This is an integrative literature review with a qualitative approach, based on the collection of information in electronic databases, using the exploratory scientific methodology. Using inclusion and exclusion criteria: Periods, languages, articles, full texts followed. Results: After filtering based on the inclusion and exclusion criteria, thirteen articles remained, responding to the objectives of the present study. From these, several impacts of the practice of physical exercises were presented on the health and quality of life of the elderly, and it was evidenced in the categories and subcategories that there are positive impacts on the mental health and on the physical health of the elderly, directly reverberating in their quality of life. life. Final Considerations: It was concluded that the benefits of the practice of assisted physical exercises are noticeable, and according to the tolerance of each elderly person, on the health and quality of life of the elderly person, and are also the main source that promotes physical and psychological well-being. and social for this audience.
\end{abstract}

Keywords: Physical exercise, Quality of life, Elderly.

\section{RESUMEN}

Objetivo: Conocer lo publicado en la comunidad científica sobre la relación entre la práctica de ejercicio físico por parte de las personas mayores y su calidad de vida y salud. Métodos: Se trata de una revisión integradora de la literatura con enfoque cualitativo, basada en la recolección de información en bases de datos electrónicas, utilizando la metodología científica exploratoria. Utilizando criterios de inclusión y exclusión: Periodos, idiomas, artículos, textos completos seguidos. Resultados: Luego de filtrar en base a los criterios de inclusión y exclusión, quedaron trece artículos, respondiendo a los objetivos del presente estudio. A partir de estos, se presentaron varios impactos de la práctica de ejercicios físicos sobre la salud y calidad de vida de los ancianos, y se evidenció en las categorías y subcategorías que existen impactos positivos en la salud mental y en la salud física de los ancianos, repercutiendo directamente en su calidad de vida. vida. Consideraciones finales: Se concluyó que los beneficios de la práctica de ejercicios físicos asistidos son notorios, y de acuerdo con la tolerancia de cada anciano, sobre la salud y calidad de vida del anciano, y son también la principal fuente que promueve el bienestar físico y psicológico. y social para esta audiencia.

Palabras clave: Ejercicio físico, Calidad de vida, Anciano.

${ }^{1}$ Faculdade Adventista da Bahia, Cachoeira - BA. *E-mail: enf.lunamoura@hotmail.com 


\section{INTRODUÇÃO}

O envelhecimento populacional (EP) se mostra como uma das mais expressivas tendências do século XXI, em países desenvolvidos bem como nos que estão em desenvolvimento. Fatores como as melhorias nas condições de saúde e sanitárias contribuíram significativamente para o aumento da expectativa de vida, tornando o envelhecimento uma das grandes evoluções para a humanidade, o que traz implicações distintas para diversos domínios da sociedade (CEPELLOS VM, et al., 2019).

O EP é um fenômeno extremamente complexo, causado por diversos fatores que produzem tendências e consequências das mais diversas. Por isso, esse fenômeno está exigindo, cada vez mais, estudos multidisciplinares para seu melhor entendimento e compreensão (JÚNIOR CSD, et al., 2006).

Há indicação do aumento da expectativa de vida, bem como do número de adultos mais velhos nos dados demográficos referentes ao Brasil nas últimas décadas. O que torna esta temática relevante é o fato de que a estimativa para os próximos 40 anos seja de que a população com 60 anos ou mais triplique em relação à população total (CEPELLOS VM, et al., 2019).

Sabe-se que o envelhecimento é um processo que provoca alterações e desgastes em vários sistemas funcionais, que acontecem de forma progressiva e irreversíveis, mas que não é um empecilho para se viver bem. Esse processo pode ser bem-sucedido quando é acompanhado de qualidade de vida e bem-estar, e para isso se faz necessário buscar e realizar algumas práticas que sejam favoráveis para obter uma qualidade de vida melhor. Uma das práticas que tem sido sugerida é a realização de exercícios físicos, sendo a inserção do mesmo na rotina de pessoas idosas fundamental, pois traz resultados quase que imediatos, ficando visíveis em curto prazo, promovendo relevantes benefícios, como, maior vigor, prevenção de diversas doenças e melhor bem-estar à medida que se envelhece (CIVINSKI C, et al., 2011).

Os efeitos positivos da prática de exercícios físicos na funcionalidade de pessoas idosas incluem maior independência em atividades de autocuidado, melhoria da autoestima, melhora qualidade de vida, maior expectativa de vida, redução do risco de quedas e da mortalidade (SCIANNI AA, et al., 2019).

Sabe-se também, que a pessoa idosa ao realizar a prática regular de exercício físico, proporciona melhora de todos os sistemas do corpo humano, desde a capacidade cognitiva e funcional, a habilidades físicas. $O$ que beneficia o idoso como um todo, pois a melhora destes sistemas o capacitam a realizar da melhor forma as atividades diárias, e consequentemente os possibilitam a viverem com qualidade. (FERRETTI F, et al., 2017).

Sobre os benefícios desta prática, sabe-se que tem papel de destaque na melhora da capacidade funcional à medida que reflete nas habilidades físicas e psíquicas, que gera uma independência para realizar as atividades de vida diária (AVDs) (GOMES NC, 2015).

Neste contexto, vale salientar um conceito que reflete de modo holístico, a mensuração do equilíbrio das áreas biopsicossociais do ser humano: a qualidade de vida. A qualidade de vida (QV) da pessoa idosa constitui um indicador de saúde essencial, sendo razoável admitir-se que todos os restantes se tornam importantes na medida em que concorrem para o bem-estar (DACA T, et al., 2016).

A QV é um fenômeno complexo, subjetivo e com múltiplos aspectos, sendo, portanto, de difícil interpretação. Envolve o julgamento individual de alguns domínios específicos da vida como autoestima e bem-estar pessoal, abrangendo aspectos relacionados à capacidade funcional, ao nível socioeconômico, estado emocional, interação social, atividade intelectual, ao autocuidado, suporte familiar, à saúde, à função sexual, aos valores culturais, éticos e religiosos, estilo de vida, satisfação com o emprego e/ou atividades diárias e ao ambiente em que se vive (SIMEÃO FFAP, et al., 2018).

Uma das abordagens sugeridas para alcançar o envelhecimento saudável e ativo é a prática regular de exercício físico. Deste modo, entende-se que a prática de exercícios físicos pode contribuir para a melhora na qualidade de vida do indivíduo (TEIXEIRA JNBJ, et al., 2016). Sendo assim, se mostra de grande relevância o estímulo à realização de atividades físicas pelas pessoas idosas de modo assistido, orientado conforme capacidades individuais. Estas práticas visam impactos na saúde e qualidade de vida destes idosos. 
Diante disso percebe-se a importância do estudo em tela, considerando o questionamento proposto dessa pesquisa: qual a relação entre as práticas de exercícios físicos por pessoas idosas e sua qualidade de vida e saúde? Logo, o estudo tem como objetivo dissertar sobre o que tem sido publicado no meio científico, sobre a relação entre as práticas de exercícios físicos por pessoas idosas e sua qualidade de vida e saúde.

\section{MÉTODOS}

Trata-se de uma revisão integrativa da literatura, tendo como base a coleta de informações nos bancos de dados eletrônicos, utilizando a metodologia cientifica exploratória. Sua finalidade é avaliar e comparar as informações dos resultados das publicações, contribuindo para o aprofundamento do conhecimento do tema sobre os benefícios que as práticas dos exercícios físicos trazem para as pessoas idosas bem como os impactos na qualidade de vida.

Para a elaboração da pesquisa utilizou-se as seguintes etapas: determinação do tema e definição da pesquisa, definição da questão da pesquisa, estabelecimento dos critérios de inclusão e exclusão (amostra), definição das categorias/informações a serem retiradas dos artigos utilizados como subsídios: autor, ano, título, revista, objetivo e desfecho.

O estudo foi realizado a partir da busca nas bases de dados Scientific Eletronic Library Online (Scielo), Biblioteca Virtual em Saúde (BVS), combinado com os descritores: Exercício físico, Qualidade de vida, Idoso, Saúde do idoso. Utilizando como recorte temporal os anos de 2014-2019 por se tratar dos artigos mais atuais publicados sobre a temática.

A sigla PICO representa a junção das primeiras letras das palavras: Paciente, Intervenção, Comparação e "Outcomes" - Desfecho (SANTOS CMC, et al., 2007). Abaixo se descreve a estratégia utilizada para a formulação da pergunta e busca de evidências, através da estratégia PICO (Quadro 1).

Quadro 1 - Descrição da aplicação da estratégia PICO, 2020.

\begin{tabular}{|c|c|c|}
\hline Iniciais & Descrição & Análise \\
\hline P & Paciente & Pessoa idosa \\
\hline I & Intervenção & Prática de exercícios físicos \\
\hline C & Comparação & $\begin{array}{r}\text { Impactos da prática/ ou não de exercícios físicos na } \\
\text { qualidade de vida e saúde da pessoa idosa. }\end{array}$ \\
\hline O & Outcomes(Desfecho) & $\begin{array}{r}\text { A relevância da prática de exercícios físicos na qualidade de } \\
\text { vida e saúde da pessoa idosa. }\end{array}$ \\
\hline
\end{tabular}

Fonte: Silva GG, et al., 2020. Baseado em Santos CMC, et al., 2007.

Como critérios de inclusão, foram aplicados: artigos publicados entre 2014-2019, disponíveis em língua portuguesa, na íntegra, e que sejam compatíveis com o objetivo do estudo.

O recorte temporal foi definido buscando os artigos mais atuais sobre a temática, e também em virtude da publicação, em 2014, da Política Nacional de Promoção à Saúde (PNPS) revisada (Brasil, et al, 2014). Neste ano foi firmada parceria entre a coordenação da PNPS e o Grupo Temático de Promoção da Saúde da Associação Brasileira de Saúde Coletiva (GT) Promoção da Saúde e Determinantes Sociais Abrasco, com mediação da Organização Pan-Americana da Saúde (OPAS). Esta política se mostra como marco na defesa dos impactos positivos dos exercícios físicos na saúde e qualidade de vida dos brasileiros, portanto se mostra interessante a análise de publicações após esta data.

Além dos critérios de inclusão citados previamente, no processo de seleção e avaliação da qualidade metodológica dos estudos foi utilizada a classificação quanto ao nível de evidência científica e quanto ao Qualis CAPES.

Os critérios de exclusão para este estudo foram: artigos repetidos, sendo considerados apenas uma vez; monografias, dissertações, teses, artigos indisponíveis na íntegra, que não atendam a todos os critérios de inclusão e que não apresentem compatibilidade com o tema após a leitura do título e resumo. 
Foi decidida pela utilização do operador booleano delimitador "AND", no intuito de restringir os achados da relação entre a prática de exercícios físicos e os impactos na qualidade de vida e saúde da pessoa idosa. $O$ resultado da busca e filtragem após os critérios é apresentando a seguir (Figura 1).

Figura 1 - Fluxograma representativo de busca na Biblioteca Virtual em Saúde.

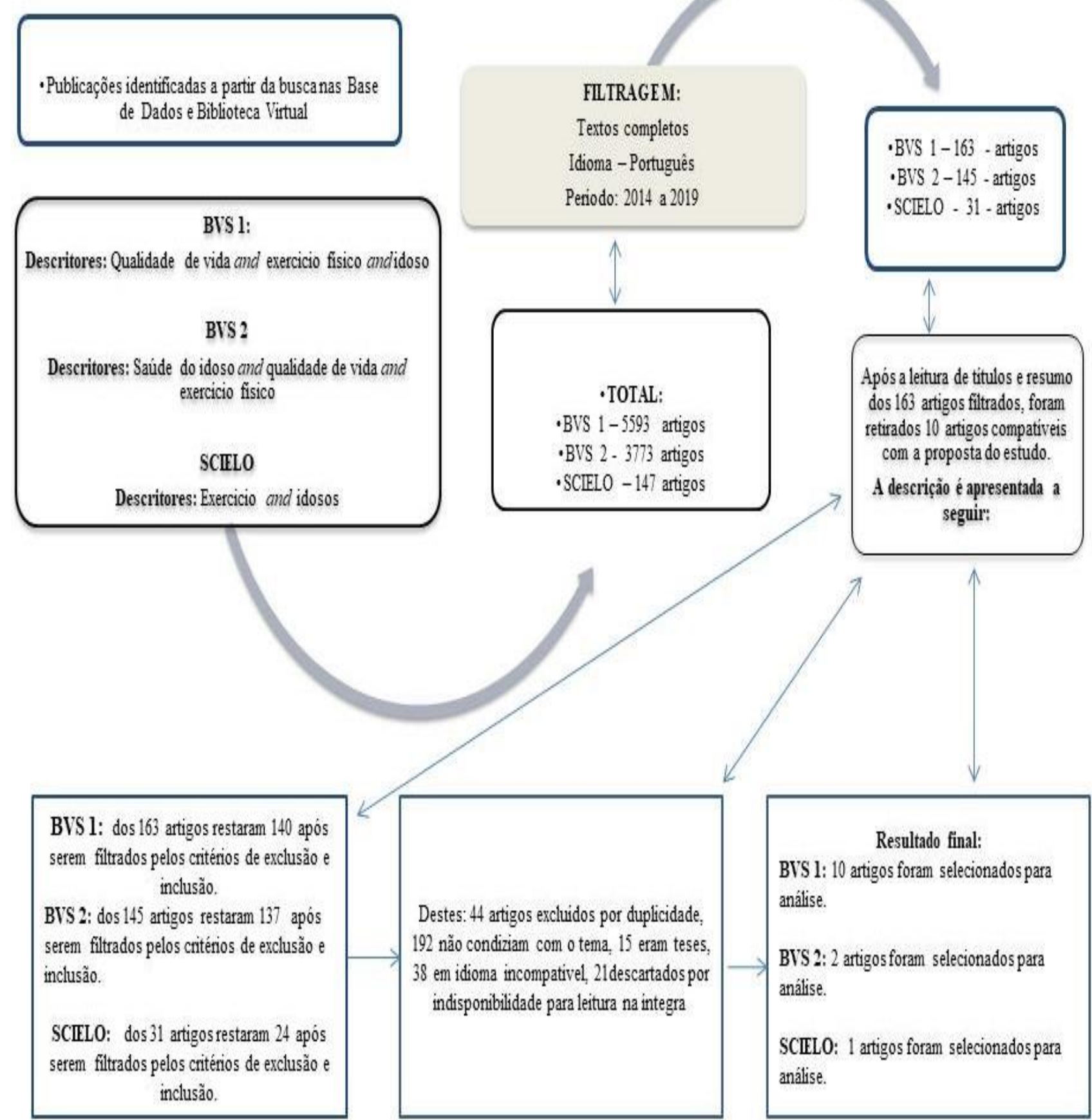

Fonte: Silva GS, et al., 2020.

Logo, ao término da busca, foram selecionados ao todo, 13 artigos para posterior análise, sendo 10 artigos da BVS contendo os descritores "Qualidade de vida and exercício físico and idoso", 2 artigos desta mesma base ao cruzar "Saúde do idoso and qualidade de vida and exercício físico" e 1 artigos da Scielo ao cruzar os descritores "Exercício físico and idosos". Na base de dados Scielo, este cruzamento se mostrou como o único a apresentar resultados após filtragem com os critérios de inclusão e exclusão. 


\section{RESULTADOS E DISCUSSÃO}

A partir da seleção de artigos feita consoante aos critérios de inclusão e exclusão, os treze manuscritos remanescentes foram dispostos abaixo, sendo apontada a fonte de busca, o termo de referência que será utilizado no estudo em tela para cada artigo (A1, A2, A3...), o nome da revista, a classificação (Qualis) Capes de cada revista, título do artigo e respectivos autores (Quadro 2).

Nos artigos selecionados, foram apresentados diversos impactos da prática de exercícios físicos na saúde e qualidade de vida da pessoa idosa. Foi apontada como fundamental a prática do exercício físico, e como principal fonte promotora do bem-estar físico, psíquico e social (SILVA AC e NEVES R, 2016). Percebeu-se também que o exercício físico favorece a saúde e melhora da qualidade de vida da população idosa (SOUZA JC, et al., 2015).

A síntese das considerações a respeito dos impactos da prática de exercícios físicos na qualidade de vida e na saúde da pessoa idosa estão apresentados no (Quadro 3). 
Quadro 2 - Quadro de artigos da mostra final, por ordem decrescente.

\begin{tabular}{|c|c|c|c|c|c|c|}
\hline Fonte & Referência & Ano & Revista & $\begin{array}{l}\text { Qualis- } \\
\text { Capes }\end{array}$ & Título & Autores \\
\hline BVS 1 & $\mathrm{~A} 1$ & 2017 & $\begin{array}{l}\text { Revista Saúde e } \\
\text { Pesquisa }\end{array}$ & B3 & $\begin{array}{l}\text { Capacidade funcional de idosos } \\
\text { institucionalizados: Revisão Integrativa. }\end{array}$ & $\begin{array}{l}\text { Temístocles Vicente Pereira Barros, Allan Derkian } \\
\text { Borges Santos, Jozilma de Medeiros Gonzaga, Maria } \\
\text { Goretti da Cunha Lisboa, Caroline Brand. }\end{array}$ \\
\hline BVS 1 & $\mathrm{~A} 2$ & 2016 & Revista Kairós & B3 & $\begin{array}{l}\text { Os olhares dos profissionais sobre os } \\
\text { programas de atividade física para pessoas } \\
\text { idosas. }\end{array}$ & Ana Catarina Silva, Rui Neves. \\
\hline BVS 1 & A3 & 2016 & $\begin{array}{l}\text { Estudos e Pesquisas em } \\
\text { Psicologia }\end{array}$ & $\mathrm{A} 2$ & $\begin{array}{l}\text { Repercussões de um programa de atenção } \\
\text { psicológica sobre a qualidade de vida de idosas } \\
\text { praticantes do método Pilates. }\end{array}$ & $\begin{array}{l}\text { Emília Cristina Ferreira de Carvalho, Marcelo de Maio } \\
\text { Nascimento. }\end{array}$ \\
\hline BVS 1 & A4 & 2015 & $\begin{array}{l}\text { Revista Saúde e } \\
\text { Pesquisa }\end{array}$ & B3 & $\begin{array}{l}\text { Qualidade de vida e indicativos de depressão } \\
\text { em idosas praticantes de exercício físico em } \\
\text { academiais da terceira idade da cidade de } \\
\text { Maringá (PR). }\end{array}$ & $\begin{array}{l}\text { Fellipe Biazin Gonçalves, Ana Paula Serra de Araújo } \\
\text { José Roberto Andrade do Nascimento Júnior, Daniel } \\
\text { Vicentini de Oliveira. }\end{array}$ \\
\hline BVS 1 & A5 & 2015 & $\begin{array}{c}\text { Estudos } \\
\begin{array}{c}\text { Interdisciplinares sobre o } \\
\text { envelhecimento }\end{array}\end{array}$ & B2 & $\begin{array}{l}\text { Análise da qualidade de vida em idosos } \\
\text { praticantes e não praticantes de exercício físico } \\
\text { regular. }\end{array}$ & $\begin{array}{l}\text { Fátima Ferretti Greyci, Christine Teló Beskow, Rosane } \\
\text { Cristina Slaviero, Cezar Grontowski Ribeiro. }\end{array}$ \\
\hline BVS 1 & A6 & 2015 & $\begin{array}{l}\text { Revista Brasileira de } \\
\text { Promoção à Saúde }\end{array}$ & B3 & $\begin{array}{l}\text { Atividade física e incapacidade funcional em } \\
\text { idosos na zona rural de um município do } \\
\text { nordeste do Brasil. }\end{array}$ & $\begin{array}{l}\text { Wisla Keile Medeiros Rodrigues, Saulo Vasconcelos } \\
\text { Rocha, Lelia Renata Carneiro Vasconcelos, Keila de } \\
\text { Oliveira Diniz. }\end{array}$ \\
\hline BVS 1 & A7 & 2015 & $\begin{array}{l}\text { Revista Psicólogo } \\
\text { InFormação }\end{array}$ & * & Qualidade de vida dos atletas da terceira idade. & $\begin{array}{l}\text { José Carlos Souza, Nicodemos Filgueira Júnio, Ceny } \\
\text { Longhi Rezende, Heloísa Bruna Grubits Freire. }\end{array}$ \\
\hline BVS 1 & A8 & 2015 & $\begin{array}{l}\text { Revista Saúde e } \\
\text { Pesquisa }\end{array}$ & B3 & $\begin{array}{l}\text { Efeitos de um programa de ginástica orientada } \\
\text { sobre os níveis de flexibilidade de idosos. }\end{array}$ & $\begin{array}{l}\text { José Leandro Soares Silva, Rosilene Maria Lucena } \\
\text { Guedes. }\end{array}$ \\
\hline BVS 1 & A9 & 2014 & $\begin{array}{l}\text { Revista Brasileira de } \\
\text { atividade física saúde }\end{array}$ & B2 & $\begin{array}{l}\text { Efeito do treinamento físico na qualidade de } \\
\text { vida em idosos com depressão maior. }\end{array}$ & $\begin{array}{l}\text { Bianca Melo, Helena Sales de Moraes, Heitor Silveira } \\
\text { Natacha Oliveira, Andrea Camaz Deslandes, Jerson } \\
\text { Laks. }\end{array}$ \\
\hline BVS 1 & A10 & 2015 & $\begin{array}{l}\text { Revista Brasileira de } \\
\text { Ciências da Saúde }\end{array}$ & B2 & $\begin{array}{l}\text { Compreensão de Idosos sobre os Benefícios da } \\
\text { Atividade Física. }\end{array}$ & $\begin{array}{l}\text { Vicente Fidélix Ferreira Gomes Júnior, Athus Bastos } \\
\text { Brandão, Francisco Jairo Medeiros de Almeida. }\end{array}$ \\
\hline BVS 2 & A11 & 2016 & $\begin{array}{l}\text { Estudos interdisciplinares } \\
\text { sobre o envelhecimento }\end{array}$ & B2 & $\begin{array}{l}\text { A influência da prática do pilates na qualidade } \\
\text { de vida dos idosos: estudo clínico e } \\
\text { randomizado. }\end{array}$ & $\begin{array}{l}\text { Fabiana Roveda Nery, Kátia Cristina Ugolini } \\
\text { Mugnol, Vivian Bertoni Xavier, Vera Lúcia dos Santos } \\
\text { Alves. }\end{array}$ \\
\hline BVS2 & A12 & 2014 & Kairós Gerontologia & B3 & $\begin{array}{l}\text { Qualidade de vida de idosos ativos e } \\
\text { insuficientemente ativos do município de Santa } \\
\text { Maria (RS). }\end{array}$ & $\begin{array}{l}\text { Caroline Silva de Freitas, Estele Caroline Welter Mereis, } \\
\text { Marisa Pereira Gonçalves. }\end{array}$ \\
\hline Scielo & A13 & 2016 & Motricidade & B1 & $\begin{array}{l}\text { Exercício físico e qualidade de vida em idosos: } \\
\text { diferentes contextos sócios comportamentais. }\end{array}$ & $\begin{array}{l}\text { Miguel Camões, Fábia Fernandes, Bruno Silva, Tiago } \\
\text { Rodrigues, Nuno Costa, Pedro Bezerra. }\end{array}$ \\
\hline
\end{tabular}

Legenda: *Apesar de se tratar de um periódico indexado, não apresenta classificação CAPES disponível.

Fonte: Silva GS, et al., 2020. 
Quadro 3 - Considerações dos impactos na qualidade de vida e na saúde da pessoa idosa, amostra final de artigos, 2020.

\begin{tabular}{|c|c|c|}
\hline Referência & Objetivo do estudo & $\begin{array}{c}\text { Considerações sobre o impacto do exercício físico na qualidade de vida e na saúde da pessoa } \\
\text { idosa }\end{array}$ \\
\hline $\mathrm{A} 1$ & $\begin{array}{l}\text { Identificar fatores relacionados à capacidade funcional de idosos } \\
\text { residentes de Instituições de Longa Permanência para Idosos } \\
\text { (ILPIs). }\end{array}$ & $\begin{array}{l}\text { Quanto aos benefícios dos exercícios físicos para os idosos, as ações multidisciplinares de exercício } \\
\text { físico são voltadas para a promoção da independência das pessoas idosas, melhorando sua qualidade } \\
\text { de vida e sua aptidão funcional, impactando na sua saúde. }\end{array}$ \\
\hline $\mathrm{A} 2$ & $\begin{array}{l}\text { Investigar os benefícios provenientes da prática de atividade física } \\
\text { como forma de aumentar a adesão das pessoas idosa a participar } \\
\text { dos programas de Atividade Física. }\end{array}$ & $\begin{array}{l}\text { A prática do exercício físico, é a principal fonte promotora do bem estar físico, psíquico e social, onde faz } \\
\text { todo o sentido que esta atividade esteja integrada num programa de saúde para idosos, e os impactos } \\
\text { favoráveis na pratica de atividade física regularmente trazem benefício ao processo de envelhecimento. }\end{array}$ \\
\hline A3 & $\begin{array}{l}\text { Apresentar a percepção da Qualidade de Vida (QV) de integrantes } \\
\text { de um Programa de Atividade Física (PAF), em caráter } \\
\text { interdisciplinar, o qual associou o método Pilates com atividades de } \\
\text { um Programa de Atenção Psicológica (PAP) }\end{array}$ & $\begin{array}{l}\text { Os idosos ao se exercitarem obtiveram melhora no seu bem-estar físico e mental, ampliando, assim, seu } \\
\text { nível/escore da qualidade de vida. Compreendendo, portanto, que os bons níveis de qualidade de vida } \\
\text { sejam fundamentais à prevenção dos fatores responsáveis pelo adoecimento e agravamento de doenças } \\
\text { pré-existentes. }\end{array}$ \\
\hline A4 & $\begin{array}{l}\text { Analisar a qualidade de vida e os indicativos de depressão em } \\
\text { idosas praticantes de exercícios físicos nas academias da terceira } \\
\text { idade atividade física do município de Maringá, Estado do Paraná. }\end{array}$ & $\begin{array}{l}\text { O exercício físico presta contribuição para favorecer a saúde e melhora da qualidade de vida da } \\
\text { população idosa. Logo, os autores apontam aspectos positivos sobre a qualidade de vida dos idosos, } \\
\text { como: a autoestima e o bem-estar pessoal, assim como o próprio estado de saúde e mudanças no estilo } \\
\text { de vida. }\end{array}$ \\
\hline A5 & $\begin{array}{l}\text { Analisar a qualidade de vida de idosos praticantes e não praticantes } \\
\text { de exercício físico regular. }\end{array}$ & $\begin{array}{l}\text { As pessoas idosas que praticam atividade física melhoram a capacidade respiratória, cardíaca, força } \\
\text { muscular, memória recente, cognição e habilidades sociais. }\end{array}$ \\
\hline A6 & $\begin{array}{l}\text { Analisar a associação entre o nível de atividade física habitual e a } \\
\text { incapacidade funcional de idosos residentes em áreas rurais }\end{array}$ & $\begin{array}{l}\text { A prática regular de atividade física é um importante fator de proteção para a incapacidade funcional } \\
\text { entre idosos, promovendo a saúde, visando e garantindo um envelhecimento saudável. }\end{array}$ \\
\hline A7 & $\begin{array}{l}\text { Avaliar a qualidade de vida de idosos praticantes de atividade física } \\
\text { regular no município de Ponta Porã }\end{array}$ & $\begin{array}{l}\text { Os autores destacam a influência positiva da atividade física, pois esta constitui um excelente } \\
\text { instrumento de promoção à saúde, melhorando suas adaptações fisiológicas e psicológicas como } \\
\text { processo de manutenção da saúde, impactando na qualidade de vida no processo de envelhecimento } \\
\text { de cada pessoa. }\end{array}$ \\
\hline A8 & $\begin{array}{l}\text { Analisar os efeitos de um programa de ginástica orientada sobre os } \\
\text { níveis de flexibilidade de idosos. }\end{array}$ & $\begin{array}{l}\text { Os exercícios de alongamento aumentam a flexibilidade, proporcionando bem-estar, força muscular e, } \\
\text { acima de tudo, bom condicionamento físico, melhorando a saúde da pessoa idosa. }\end{array}$ \\
\hline A9 & $\begin{array}{l}\text { Comparar diferentes intervenções com exercícios físicos na } \\
\text { qualidade de vida e nos sintomas depressivos em idosos } \\
\text { depressivos. }\end{array}$ & $\begin{array}{l}\text { Os efeitos positivos das práticas dos exercícios físico na saúde dos idosos, impactam em melhora na } \\
\text { qualidade de vida, e com isso os autores perceberam que foram reduzidos os sintomas depressivos em } \\
\text { pessoas idosas. }\end{array}$ \\
\hline A10 & $\begin{array}{l}\text { Analisar como um grupo de idosos praticantes de exercícios físicos } \\
\text { compreendem os efeitos proporcionados pela pratica física. }\end{array}$ & $\begin{array}{l}\text { A atividade física consegue promover a longevidade, melhora a cognição, o estado mental e os níveis de } \\
\text { humor, melhorando a saúde dos idosos. }\end{array}$ \\
\hline A11 & $\begin{array}{l}\text { Verificar o impacto de um protocolo de exercício físico focado no } \\
\text { método Pilates na qualidade de vida de idosos. }\end{array}$ & $\begin{array}{l}\text { A prática de exercícios regular e sistematizada tem papel fundamental na proteção da capacidade } \\
\text { funcional dos idosos, os benefícios e os protocolos de exercício em idosos também têm influenciado na } \\
\text { qualidade de vida e na manutenção da capacidade funcional, gera impacto positivo na autonomia dos } \\
\text { idosos, dentre outros fatores. }\end{array}$ \\
\hline A12 & $\begin{array}{l}\text { Analisar a qualidade de vida de idosos pertencentes a grupos de } \\
\text { convivência ativos e de idosos insuficientemente ativos, não } \\
\text { pertencentes a grupos de convivência. }\end{array}$ & $\begin{array}{l}\text { A atividade física regular vem sendo praticada com o objetivo de manter saúde dos idosos, e com isso } \\
\text { prevenindo algumas doenças e mortes prematuras, percebe-se que os idosos fisicamente ativos } \\
\text { demonstraram melhor desempenho nos diferentes domínios da qualidade de vida. }\end{array}$ \\
\hline A13 & $\begin{array}{l}\text { Descrever a percepção da qualidade de vida em indivíduos acima } \\
\text { dos } 70 \text { anos, tendo em conta a participação em programas de } \\
\text { exercício físico em contextos comunitários e idosos } \\
\text { institucionalizados. }\end{array}$ & $\begin{array}{l}\text { A estratégias de prevenção e tratamento com base no exercício físico, devem ser privilegiadas em todos } \\
\text { os contextos sócio comportamentais, como forma de manter a autonomia e consequentemente a } \\
\text { qualidade de vida, entre a população envelhecida. }\end{array}$ \\
\hline
\end{tabular}

Fonte: Silva GS, et al., 2020. 
A partir da leitura dos artigos em tela, emergiram 2 categorias: "impactos da prática de exercício físico na saúde da pessoa idosa" e "impactos da prática de exercício físico na qualidade de vida da pessoa idosa". A primeira categoria foi subdividida em 2 subcategorias: "impactos na saúde física da pessoa idosa" e "impactos na saúde mental da pessoa idosa".

Neste contexto, apesar do envelhecimento ser um fator inevitável, o declínio fisiológico varia consideravelmente entre os indivíduos; sendo que as perdas inerentes ao envelhecimento natural podem ser minimizadas com a prática de exercício físico (CAMÕES M, et al., 2016). Dito isto, outros estudos corroboram que os impactos favoráveis na prática de atividade física regularmente são de benefícios para um processo de envelhecimento saudável (SILVA AC e NEVES R, 2016). Ficou destacado entre os estudos, que o exercício físico continuado em idosos aprimora a capacidade respiratória, frequência cardíaca, força muscular, memória recente, cognição e habilidades sociais, além dos níveis de aptidão física e capacidade funcional, que são aspectos importantes para uma saúde de qualidade (FERRETTI F, et al., 2017).

Desta forma destaca-se os benefícios da prática do exercício físico na saúde mental da pessoa idosa. Sendo assim, pesquisadores concluíram que as idosas praticantes de exercícios físicos possuem um bom nível de qualidade de vida geral, além de baixos índices de indicativos de depressão (GONÇALVES FB, et al., 2015). Foram ressaltados também em outras pesquisas, os impactos das práticas dos exercícios físicos na saúde dos idosos, pontuando que com isso reduziram-se os sintomas depressivos (MELO B, et al., 2014).

Outros estudos pontuaram que estas atividades melhoram a qualidade de vida e conseguem promover a longevidade, melhora a cognição, o estado mental e os níveis de humor; impactando positivamente na saúde mental dos idosos (JÚNIOR FFG, et al., 2015). Referente aos impactos do exercício físico na saúde física de pessoas idosas, percebeu-se que idosos ao se exercitarem obtiveram melhora no seu bem-estar físico e mental, ampliando assim seus níveis de qualidade de vida (CARVALHO RBC e MADRUGA VA, 2010).

Foi apresentado também que, mediante a prática do exercício físico a pessoa idosa pode ampliar seus níveis de força muscular, o fluxo sanguíneo, reduzindo o percentual de gordura corporal e de glicose, baixando os níveis da pressão arterial, além de melhorar a densidade corporal óssea (CARVALHO ECF e NASCIMENTO MM, 2016). Levando em consideração que o processo de envelhecer pode gerar limitações funcionais, afetando a QV, foi evidenciado que o exercício físico é um método eficaz para atenuar os processos de declínio observados durante o envelhecimento, conservando sua capacidade funcional e qualidade de vida em boas condições (FERRETTI F, et al., 2017).

Entende-se que a qualidade de vida durante o processo do envelhecimento está voltada não apenas a aspectos físicos, mas o idoso como um todo, está associada a autoestima, ao estado de saúde mental, ao estilo de vida. A partir desta perspectiva a adoção de um estilo de vida ativo e saudável, é fundamental para a promoção de um envelhecer com qualidade, sendo o exercício físico uma das fundamentais praticas que contribui de forma direta na promoção de saúde e de bem-estar físico, mental e cognitivo, promovendo a longevidade, melhora nos níveis de humor, o que resulta na redução de depressão, isolamento e outros transtornos, dessa forma se torna um fator determinante na senescência e para a promoção de qualidade de vida durante esse processo (MAIA RR e RACHED CDA, 2017).

O ambiente em grupo de exercícios, além de benefícios orgânicos e físicos, proporciona uma melhor socialização e distanciamento de agravos psicológicos, interferindo diretamente na redução de isolamento, depressão e autoestima baixa. Os benefícios da atividade física, proporcionam liberdade de locomoção, interação social e lazer, nessa perspectiva, pode-se inferir que a prática de exercício físico, sobretudo quando é feita através de grupos, funciona como um espaço de promoção do papel social do idoso, permitindo a manutenção da sua autonomia e estimulando a construção da autoestima, muitas vezes esquecida durante o processo de envelhecimento (GOMES NC, 2015).

Portanto, mediante o exposto, a atividade física regular tem reflexos positivos sobre a melhoria da capacidade funcional e as habilidades físicas em indivíduos idosos, auxiliando a reduzir as taxas de dependência para realização de suas AVDs, aspecto físico débil e declínio em sua esfera social, promovendo a interação e desenvolvimento de um estilo de vida mais saudável, deixando-o fisicamente ativo e melhorando sua qualidade de vida (JÚNIOR FFG, et al., 2015). 
Vale salientar, sobre os impactos da prática de exercício físico na qualidade de vida da pessoa idosa, que os estudos apontaram que os exercícios de alongamento aumentam a flexibilidade, proporcionando bemestar, força muscular e, acima de tudo, bom condicionamento físico, melhorando a saúde da pessoa idosa (SILVA JLSJ e GUEDES RML, 2015). Outros corroboram, portanto, que a prática regular de atividade física é um importante fator de proteção para a incapacidade funcional entre idosos, sendo assim minimizando a degeneração provocada pelo envelhecimento e com isso melhoram na qualidade de vida (RODRIGUES WKM, et al., 2015).

De modo geral, foi percebido então, que o envelhecimento ativo se mostra como um fator primordial para o prolongamento da vida com qualidade para idosos, uma vez que o mesmo proporciona o afastamento da dependência funcional e de relacionadas ao estado psicossocial do indivíduo, como depressão e isolamento, que conduzem a uma vida sedentária, predispondo ao surgimento de doenças cardiovasculares e de outras disfunções sistêmicas (JÚNIOR FFG, et al., 2015). Logo, iniciativas que envolvam as pessoas idosas em atividades físicas e relações sociais, são benéficas para sua qualidade de vida.

Foi apresentado nos estudos selecionados, que QV é um fenômeno complexo, subjetivo e com múltiplos aspectos, sendo, portanto, de difícil interpretação. Envolve o julgamento individual de alguns domínios específicos da vida como autoestima e bem-estar pessoal, abrangendo aspectos relacionados à capacidade funcional, ao nível socioeconômico, estado emocional, interação social, atividade intelectual, ao autocuidado, suporte familiar, à saúde, à função sexual, aos valores culturais, éticos e religiosos, estilo de vida, satisfação com o emprego e/ou atividades diárias e ao ambiente em que se vive (SIMEÃO SFAP, et al., 2018).

Neste contexto, os artigos selecionados apresentam que a QV um conceito que reflete de modo holístico, a mensuração do equilíbrio das áreas biopsicossociais do ser humano. A QV da pessoa idosa constitui um indicador de saúde essencial, sendo razoável admitir-se que todos os restantes se tornam importantes na medida em que concorrem para o bem-estar (DACA T, et al., 2016).

Foi apresentado também, que qualidade de vida é percebida pelo indivíduo como sua posição na vida, no contexto da cultura e sistema de valores, nos quais ele relaciona seus objetivos, expectativas, padrões e preocupações (FREITAS CS, et al., 2014). Sendo assim, quando se fala em qualidade de vida da pessoa idosa, aspectos como o exercício físico, prestam grande contribuição para favorecer a saúde e melhora da qualidade de vida da população idosa. Logo, os estudos apontaram aspectos positivos sobre a qualidade de vida dos idosos, como: a melhora na autoestima e o bem-estar pessoal, assim como o próprio estado de saúde e o estilo de vida.

O exercício físico configura-se como uma importante intervenção não farmacológica para a população idosa, principalmente quando se trata de doenças que possui como fator causante o sedentarismo, pois reduz os níveis pressóricos e auxilia na melhora da resposta do organismo. Reduzindo assim a amplitude da polifarmácia, os riscos de interações medicamentosas e os efeitos sistêmicos das grandes doses de medicamentos. Sendo então um meio de prevenção e tratamento de diversas doenças, além de promover bem-estar e longevidade com qualidade (GOMES NC, 2015).

Um dos artigos selecionados apresenta os principais objetivos de um Programa de Atividade Física (PAF) disponibilizado em diferentes instituições. Como os PAF para idosos objetivam a promoção da saúde para os seus utentes, é-lhes fundamental que os recursos humanos e os recursos físicos sejam qualificados e adequados para esse efeito (SILVA AC e NEVES R, 2016). Ainda sobre como a atividade física influenciou na vida de idosos, foi observado um impacto positivo sobre a aptidão funcional, socialização, aumento da autoestima. Apresentam o relato de uma idosa: "Sinto que melhorou as dores, me sinto mais vigorosa". Consigo realizar melhor minhas atividades, mais disposta e me sinto bem comigo mesma. Outra idosa pontuou neste artigo: "Aumentou minha disposição e diminuiu a quantidade de remédio para a pressão e para dor que eu tomava” (JÚNIOR FFG, et al., 2015).

Logo, a partir do exposto, os impactos positivos da prática de exercícios físicos acompanhados e conforme tolerância de cada idosos, são expressivos. Foram apresentados impactos positivos que influenciam diretamente na qualidade de vida das pessoas idosas e em seu desempenho das atividades básicas e instrumentais de vida diária. 


\section{CONSIDERAÇÕES FINAIS}

Conclui-se que são perceptíveis os benefícios da prática de exercícios físicos assistidos e conforme a tolerância de cada idoso. Foi evidenciado que há impactos positivos na saúde mental e na saúde física das pessoas idosas, reverberando diretamente na qualidade de vida destas pessoas idosas. Foi expressivo que estas práticas de exercícios físicos ajudam nas atividades da vida diária que eles venham desenvolver, e na funcionalidade das pessoas idosas holisticamente. Destaca-se, por fim, a relevância do desenvolvimento de mais estudos com essa temática, elucidando os efeitos positivos da prática do exercício físico na saúde mental e física da pessoa idosa. Sugere-se que ensaios clínicos sejam realizados para corroborar ou confrontar com os diversos achados qualitativos que estão postos na comunidade científica acerca da temática.

\section{REFERÊNCIAS}

1. BARROS TVP, et al. Capacidade funcional de idosos institucionalizados: revisão integrativa. Revista Kairós Gerontologia, 2016; 41(3): 176-180.

2. BRASIL. Ministério da Saúde. Política Nacional de Promoção da Saúde. PNaPS: Revisão da Portaria MS/GM n 687, de 30 de março de 2006. Brasília, 2014.

3. CAMÕES M, et al. Exercício físico e qualidade de vida em idosos: diferentes contextos sociocomportamentais. Motricidade, 2016; 12(1): 96-105.

4. CARVALHO ECF, NASCIMENTO MM. Repercussões de um programa de atenção psicológica sobre a qualidade de vida de idosas praticantes do método Pilates. Estudos e Pesquisas em Psicologia; 2016; 16(2): 488-507.

5. CARVALHO RBC, MADRUGA VA. Envelhecimento e prática de atividade física: a influência do gênero. Motriz: Revista de Educação Física, 2010; 17(2): 328.

6. CEPELLOS VM, et al. Envelhecimento: múltiplas idades na construção da idade profissional. Organizações \& Sociedade, 2019; 26(89): 269-290.

7. CIVINSK C, et al. A importância do exercício físico no envelhecimento. Revista da Unifebe, 2011; 163-175.

8. DACA T, et al. Exercício físico e saúde para o idoso em Moçambique. Rev. cient. UEM: Sér. ciênc. bioméd. saúde pública, 2016; 1(2): 23-32.

9. FERRETTI F, et al. Análise da qualidade de vida em idosos praticantes e não praticantes de exercício físico regular. Estudos Interdisciplinares sobre o Envelhecimento, 2017; 20(3): 729-743.

10. FREITAS CS, et al. Qualidade de vida de idosos ativos e insuficientemente ativos do município de Santa Maria (RS). Revista Kairós Gerontologia, 2014; 17(1): 57-68.

11. GOMES NC. Farmacoterapia e fatores associados à adesão ao tratamento em idosos com síndrome metabólica. 2015. 166f. Dissertação (Mestrado em Atenção à Saúde) - Universidade Federal do Triângulo Mineiro, Uberaba, Minas Gerais, Brasil, 2015.

12. GONÇALVES FB, et al. Qualidade de vida e indicativos de depressão em idosas praticantes de exercícios físicos em açademias da terceira idade da cidade de Maringá. Saúde e Pesquisa, 2015; 8(3): 23-39.

13. JÚNIOR CSD, et al. O envelhecimento da população brasileira: uma análise de conteúdo das páginas da REBEP. Revista brasileira de geriatria e Gerontologia, 2006; 9(2): 7-24.

14. JÚNIOR FFG, et al. Compreensão de idosos sobre os benefícios da atividade física. Revista Brasileira De Ciências Da Saúde, 2015; 19(3): 193-198.

15. MAIA RR, RACHED CDA. Atividade física: a atuação do enfermeiro para a promoção da saúde na terceira idade-uma revisão de literatura. International Journal Of Health Management Review, 2017; 3(1).

16. MELO B, et al. Efeito do treinamento físico na qualidade de vida em idosos com depressão maior. Revista Brasileira de Atividade Física \& Saúde, 2014; 19(2): 205-214.

17. NERY FR, et al. A influência da prática do pilates na qualidade de vida dos idosos: Estudo clínico e randomizado. Estudos Interdisciplinares sobre o Envelhecimento, 2017; 21(2): 75-88.

18. RODRIGUES WKM, et al. Atividade física e incapacidade funcional em idosos da zona rural de um município do nordeste do Brasil. Revista Brasileira Promoção Saúde, 2015; 28(1): 126-132.

19. SANTOS CMC, et al. The PICO strategy for the research question construction and evidence search. Rev LatinoamEnfermagem, 2007; 15(3): 508-11.

20. SCIANNI AA, et al. Efeitos do exercício físico no sistema nervoso do indivíduo idoso e suas consequências funcionais. Revista Brasileira de Ciências do Esporte, 2019; 41(1): 81-95.

21. SILVA AC, NEVES R. Os olhares dos profissionais sobre os programas de atividade física para pessoas idosas. Revista Kairós Gerontologia, 2016; 19(2): 23-39.

22. SILVA AC, NEVES R. Programas de atividade física para pessoas idosas institucionalizadas - contextos e práticas. Revista Kairós Gerontologia, 2017; 20(4): 09-25.

23. SILVA JLSJ, GUEDES RML. Efeitos de um programa de ginástica orientada sobre os níveis de flexibilidade de idosos. Saúde e Pesquisa, 2015; 8(3): 541-548.

24. SIMEÃO SFAP, et al. Estudo comparativo da qualidade de vida de idosos asilados e frequentadores do centro dia. Ciência \& saúde Coletiva, 2018; 23(11): 3923-3934.

25. SOUZA JC, et al. Qualidade de vida dos atletas da terceira idade. Psicólogo InFormação; 2015; $19(19)$ : $176-180$.

26. TEIXEIRA JNBJ, et al. Exercício físico na atenção primária: comparação da presença de sintomas depressivos, risco de quedas e qualidade de vida entre idosos ativos e sedentários. Revista CPAQV-Centro de Pesquisas Avançadas em Qualidade de Vida-CPAQV, 2016; 11(1). 